\title{
Resultados de la vigilancia centinela de gastroenteritis por rotavirus en Chile
}

\author{
Janepsy Díaz T., Andrea Olea N., Miguel O’Ryan G., Nora Mamani M., Héctor Galeno A. y Judith Mora R.
}

\section{Sentry surveillance study of rotavirus gastroenteritis in Chile}

A sentinel-based outpatient and inpatient surveillance for rotavirus infection has been implemented in Chile. Aim: Update the impact of rotavirus infections in Chile. Methods: Ambulatory surveillance is performed in 14 centers from 8 Regions and hospital-based surveillance in 8 hospitals from three Regions (V, VIII and Metropolitan). Results: In 2007, 339 stool samples that represented 9.3\% of all outpatient cases were studied of which $15 \%$ were rotavirus positive. A total of 2.074 children younger than 5 years of age were hospitalized representing $9 \%$ of all hospitalizations for this age group. Rotavirus was detected in $13.6 \%$ of these cases. Conclusions: In the current epidemiological situation, rotavirus surveillance needs to be sustained with increased efforts to detect cases in order to avoid underreporting. Serotype/genotype surveillance of rotavirus strains needs to be included in the near future.

Key words: Rotavirus surveillance, sentry centres.

Palabras clave: Vigilancia epidemiológica, centinela rotavirus.
Ministerio de Salud de Chile División de Planificación Sanitaria Departamento de Epidemiología Unidad de Vigilancia (JDT, AON) Universidad de Chile Facultad de Medicina Instituto de Ciencias Biomédicas Programa de Microbiología (MO'RG, NMM)

Instituto de Salud Pública de Chile Laboratorio de Virología (HGA, JMR)

Recibido: 3 marzo 2008 Aceptado: 16 septiembre 2008

\section{Correspondencia a:} Janepsy Díaz Tito janepsy@hotmail.com

\section{Introducción}

$\mathrm{L}$ as enfermedades diarreicas agudas son una de las patologías que causan mayor morbilidad y mortalidad en los niños bajo 5 años de edad. Diversos agentes etiológicos infecciosos pueden producir estos cuadros, entre ellos los agentes virales, siendo rotavirus una de las más importantes causas de diarrea moderada a grave y el responsable de gastroenteritis y deshidratación grave en niños pequeños de países industrializados y en vías de desarrollo. La manifestación de una infección por rotavirus varía desde una forma asintomática hasta casos graves de vómitos y diarreas que pueden llevar a la deshidratación, shock y muerte ${ }^{1}$.

Estudios publicados entre 1986 y 1999 indican que rotavirus es el causante de $\sim 22 \%$ de las hospitalizaciones por diarreas, informándose mundialmente alrededor de 111 millones de casos y 600.000 muertes anuales; $95 \%$ de estos niños son infectados entre los 1 y 3 años de edad. En los últimos años, la infección por rotavirus se ha incrementado y se estima actualmente como el responsable de $39 \%$ de las hospitalizaciones de diarrea en niños bajo 5 años de edad (rango: 29$45 \%$ ) y el causante aproximadamente de 611.000 muertes por año. De los datos disponibles en la región de las América, rotavirus causa aproximadamente 75.000 hospitalizaciones y cerca de 15.000 muertes anuales ${ }^{1,2}$.
En Chile, "gastroenteritis por rotavirus" es un diagnóstico que se ha incrementado en el transcurso de los años, representando $13 \%$ del total de egresos hospitalarios* por diarrea en los niños bajo 5 años de edad en el año 2001 y $16 \%$ para los años 2003 y 2004. Los datos aportados por la vigilancia de centros centinela ambulatorios en el año 2006, comprobaron una positividad de $13 \%$ a este agente en un total de 1.240 muestras estudiadas durante doce meses, con una proporción similar en ambos sexos. Se observó un mayor porcentaje de positividad bajo 2 años de edad ${ }^{3}$.

Según estudios realizados por uno de los autores (M. O`Ryan y cols), rotavirus es la causa más frecuente de síndrome diarreico agudo (SDA) bajo 3 años de edad, motivando cerca de $50 \%$ de las hospitalizaciones y $35 \%$ de las consultas de urgencia. Se estima por ello, que anualmente en este grupo etario, cerca de 58.000 y 8.000 niños bajo 3 años de edad requieren una consulta de urgencia u hospitalización, respectivamente, a causa de esta infección ${ }^{4}$.

Por ello, una de las líneas de trabajo del Departamento de Epidemiología del Ministerio de Salud de Chile, a partir del año 2002, ha sido la vigilancia centinela de diarrea en niños bajo 5 años de edad. Desde el 2004, en conjunto con la Universidad de Chile, se

\footnotetext{
* Base de Egresos Hospitalarios. Departamento de Estadística e Información de Salud DEIS. MINSAL.
} 
realiza el diagnóstico de laboratorio de este agente para los casos ocurridos en niños bajo 5 años de edad atendidos en forma ambulatoria. A partir del año 2007 se estableció la vigilancia en hospitales centinelas, de notificación "en línea" con el apoyo del Instituto de Salud Pública (ISP), donde se realiza él diagnóstico de laboratorio de este agente para los casos detectados en el ámbito hospitalario.

La vigilancia a través de centros centinelas hospitalarios obedece a una propuesta de vigilancia planteada por la Organización Panamericana de la Salud (OPS), para que sea implementada por los países de la región. Se debe utilizar una muestra representativa de la población en riesgo, que permita estimar la prevalencia o incidencia del problema estudiado, a través de la información entregada por los centros informantes ${ }^{1,5}$.

Objetivos. Conocer la epidemiología de las diarreas causadas por rotavirus en Chile. Evaluar la carga de morbilidad y mortalidad de la enfermedad.

\section{Material y Método}

El protocolo del sistema de vigilancia centinela $\mathrm{am}$ bulatorio de diarrea aguda en niños bajo 5 años de edad se encuentra implementado en las 13 regiones administrativas del país, con 34 centros involucrados registran todos los casos que consultan por diarrea aguda y son pacientes bajo 5 años de edad- de éstos, 14 centros ubicados en 8 de las 13 regiones, son encargados de realizar la vigilancia etiológica para rotavirus, basados en la siguiente definición de caso: Casos nuevos ( $1^{a}$ consulta) con evacuación de heces acuosas tres o más veces en 24 horas, con o sin deshidratación, en niños bajo 5 años de edad, hasta 3 días de evolución.

Dichas muestras de deposiciones son analizadas en el Instituto de Ciencias Biomédicas, de la Facultad de Medicina, de la Universidad de Chile. Esta vigilancia abarca el $52 \%$ de la población bajo vigilancia de morbilidad en centros centinelas bajo 5 años de edad**. Las regiones en las que se encuentra implementada esta vigilancia fueron: Arica, Antofagasta, Coquimbo, Valparaíso (S.S. Viña del Mar, S.S. Quillota y S.S. Aconcagua), Metropolitana, Bío-Bío (S.S. Ñuble, S.S. Talcahuano y S.S. Bío-Bío), la Araucanía (S.S. Araucanía Sur) y Aysén.

Para la vigilancia centinela hospitalaria, se seleccionó tres regiones de Chile (Metropolitana, Bío-Bío y Valparaíso). Se aplicó el protocolo de vigilancia en ocho hospitales pediátricos, que representan una cobertura asistencial hospitalaria de $62 \%$ de la población bajo 5 años de edad en el país***, utilizando la si-

** Población inscrita en Centros Centinelas Ambulatorios 51.387. *** Población INE Menor de cinco años 767.651 guiente definición de caso: Todo niño bajo 5 años de edad, hospitalizado por diarrea aguda que tiene una muestra de heces oportuna (tomada hasta 48 horas del ingreso hospitalario). Los centros involucrados en esta vigilancia por región fueron: Valparaíso: H. Dr. Carlos Van Buren, H. Dr. Claudio Vicuña, H. Dr. Gustavo Fricke. Metropolitana: H. Dr. Luis Calvo Mackenna, H. Exequiel González Cortés, H. Dr. Félix Bulnes Cerda. Bío Bío: H. Dr. Víctor Ríos Ruiz y H. San José de Coronel.

Las muestras de deposiciones fueron analizadas en el ISP. Tanto el ISP como el laboratorio de la Universidad de Chile que desarrollan la vigilancia emplean para el análisis de las muestras la técnica inmunoenzimática IDEIA-Rotavirus (OXOID).

El análisis de los datos obtenidos mediante la vigilancia se procesó en el programa estadístico epinfo 2000 , en forma semanal, quincenal y mensual a cada nivel correspondiente según las necesidades, es decir, si existiera brotes esto se hacía en forma diaria y las acciones de focos serían inmediatas, por constituir una alarma epidemiológica. De comportarse como una situación habitual, la información se analizó semanalmente de forma preliminar y quincenal con información definitiva, con el objetivo de conocer el comportamiento de la enfermedad y monitorear el sistema de vigilancia.

\section{Resultados}

Se realizó un análisis de la vigilancia correspondiente al año 2007, tanto ambulatoria como hospitalaria.

Vigilancia en centros centinelas ambulatorios. El número de casos con diarrea en niños bajo 5 años, reportado por los centros centinelas fue de 3.629 casos (tasa de incidencia 70,6 por mil hab). De ellos, 339 casos (tasa de 6,6 por mil hab) cumplían con la definición de caso para estudio etiológico de rotavirus, representando el 9,3\% del total de casos. De las muestras estudiadas 51 fueron positivas a rotavirus (tasa de 1,0 por mil hab), alcanzando $15 \%$ positividad con relación al total de las muestras tomadas. En globo, de los 3.629 casos de diarreas diagnosticados en el ámbito ambulatorio, sólo 1,4\% fue causado por rotavirus. La región que presentó la tasa de incidencia más alta fue Antofagasta (4,6 por mil hab): $15,9 \%$. (Tabla 1). El grupo de lactantes fue el más afectado alcanzando $86,7 \%$ de positividad. El sexo femenino concentró el $56,3 \%$ de positividad.

Vigilancia en centros centinelas hospitalarios. El total de hospitalizaciones en niños bajo 5 años por todas las causas, fue de 21.880 casos. Las internaciones por diarrea fueron 2.074 , lo que corresponde a $9 \%$ del 


\begin{tabular}{|c|c|c|c|c|c|c|}
\hline Región & $\begin{array}{l}\text { Autoridades sanitarias } \\
\text { locales }\end{array}$ & Centros centinelas & $\begin{array}{l}N^{\circ} \text { casos con } \\
\text { diarrea }\end{array}$ & $\begin{array}{c}\mathrm{N}^{\circ} \text { total de } \\
\text { muestras tomadas }\end{array}$ & $\begin{array}{l}\text { No total de exámenes } \\
\text { positivos a rotavirus }\end{array}$ & $\%$ \\
\hline I & Arica & Consultorio Dr. Amador Neghme & 534 & 25 & 8 & 32,0 \\
\hline 11 & Antofagasta & Consultorio Juan Pablo Segundo & 133 & 88 & 14 & 15,9 \\
\hline IV & Coquimbo & Consultorio Tierras Blancas & 197 & 0 & 0 & \\
\hline \multirow[t]{2}{*}{ v } & Viña del Mar - Quillota & Consultorio Eduardo Frei $\mathrm{M}$. & 90 & 87 & 13 & 13,9 \\
\hline & Aconcagua & Consultorio General Urbano San Felipe & 530 & 28 & 3 & \\
\hline \multirow[t]{4}{*}{ RM } & Metropolitano Central & Consultorio de Maipú & 601 & 0 & 0 & 9,0 \\
\hline & Metropolitano Sur & Consultorio San Bernardo & 304 & 8 & 0 & \\
\hline & Metropolitano Sur Oriente & Consultorio Villa O'Higgins & 224 & 27 & 3 & \\
\hline & Metropolitano Oriente & Consultorio Dr. Aníbal Ariztía A. & 176 & 9 & 1 & \\
\hline \multirow[t]{3}{*}{ VIII } & Ñuble & Consultorio Violeta Parra & 334 & 48 & 7 & 13,4 \\
\hline & Talcahuano & Centro de Salud San Vicente & 174 & 19 & 2 & \\
\hline & Bío Bío & Consultorio Norte & 159 & 0 & 0 & \\
\hline IX & Araucanía Sur & Centro de Familia Amanecer & 43 & 0 & 0 & \\
\hline$x I$ & Aysén & Consultorio Alejandro Gutiérrez & 130 & 0 & 0 & \\
\hline Total & & & 3.629 & 339 & 51 & 15,0 \\
\hline
\end{tabular}

\begin{tabular}{|c|c|c|c|c|c|}
\hline Regióı & & Hospitales centinelas & $N^{\circ}$ total de muestras & $\mathbf{N}^{\circ}$ total de exámenes positivos & $\%$ \\
\hline \multirow[t]{3}{*}{ V } & Valparaíso & Dr. Carlos Van Buren & 98 & 17 & 23,9 \\
\hline & & Gustavo Fricke & 30 & 9 & \\
\hline & & Dr. Claudio Vicuña & 19 & 8 & \\
\hline \multirow[t]{3}{*}{ RM } & Metropolitana & Dr. Luis Calvo Mackenna & 34 & 19 & 41,3 \\
\hline & & Dr. Exequiel Gonzalez Cortés & 161 & 62 & \\
\hline & & Dr. Félix Bulnes Cerda & 8 & 0 & \\
\hline \multirow[t]{2}{*}{ VIII } & Bío -Bío & Dr. Víctor Ríos Ruiz & 414 & 86 & 25,4 \\
\hline & & San José de Coronel & 301 & 82 & \\
\hline Total & & & 1.065 & 283 & 26,6 \\
\hline
\end{tabular}

total de las hospitalizaciones (tasa 2,7 por mil hab). Del total de menores ingresados por diarreas, 1.065 cumplieron con el criterio de caso sospechoso, con muestras de heces colectadas oportunamente y estudiadas, lo que representa al 51,3\% de los niños hospitalizados por diarrea, (tasa 1,4 por mil hab). De estas muestras, 283 fueron positivas a rotavirus $(26,6 \%$ de positividad). Ello significa, que $13,6 \%$ de los niños bajo 5 años internados por diarrea padecen de rotavirosis.

La región con la tasa de incidencia más alta fue la Región del Bío-Bío, (tasa 1,2 por mil hab), con una positividad de $25,4 \%$ (Tabla 2 ).

El $60 \%$ del total de casos eran de sexo masculino y los lactantes representaron el $65 \%$ de los casos.

\section{Conclusiones}

En Chile, las condiciones sanitarias y nutricionales actuales han permitido que las enfermedades diarreicas no figuren entre las cuatro principales causas de muertes en el niño bajo 5 años de edad; sin embargo, persiste como una patología de alta frecuencia ${ }^{6,7}$. Por ello realizamos la vigilancia de diarrea en niños bajo 5 años de edad en la modalidad de vigilancia centinelas, según lo establece el Reglamento vigente sobre Notificación de Enfermedades Transmisibles de Declaración Obligatoria (DS No 158). Este sistema nos permite conocer cómo se comporta esta patología y tener un conocimiento de la epidemiología de rotavirus en el 
país, además de cumplir con la orientación de la Organización Mundial de la Salud (OMS) y la OPS respecto a la contribución de los diferentes países al sistema de vigilancia de la región.

En nuestro país las hospitalizaciones debidas a gastroenteritis por rotavirus entre los años 2001 y 2005 correspondieron a $16 \%$ de los egresos por diarrea, rango: (12-20\%), información obtenida mediante el análisis de la base de egresos hospitalarios. Además, no se registran muertes por esta causa en la actualidad. La vigilancia hospitalaria del año 2007 arrojó resultados similares, del total de las causas de las hospitalizaciones por diarrea en niños bajo 5 años de edad, 13,6\% correspondió a rotavirus. Esta cifra es inferior a lo observado en otros países de América: Paraguay y Bolivia (43,0\%), Guatemala ( $>80 \%)$. Si bien los datos nacionales muestran resultados mejores al resto de los países de la región, cabe señalar que en América Latina, Chile es el país que cuenta con mayor cantidad de hospitales involucrados en la vigilancia, donde se usa la definición de caso establecida en la Circular $\mathrm{N}^{\circ} \mathrm{B} 51$ No35 del 22 de septiembre de 2006.

Sin embargo, debemos considerar que la vigilancia centinela se realiza sólo con una muestra representativa de la población, y que dada la situación epidemiológica actual de rotavirus en el mundo, es importante mantener este sistema de vigilancia, ampliar la pesquisa actual de los casos, aplicar el protocolo de vigilancia establecido y, con el apoyo de los laboratorios involucrados, determinar el serotipo/genotipo circu- lante en el país. Es importante considerar que para obtener la prevalencia real de rotavirus en el país, debemos realizar estudios de prevalencia como complemento de la vigilancia.

La información obtenida nos permitirá contribuir en forma dinámica y garantizar una correcta toma de decisión sobre bases científicas en todos los niveles del Sistema de Salud. De esta manera, los resultados obtenidos a través del sistema serán de gran importancia para Chile y experiencia para otros países de la región.

\section{Resumen}

En Chile, se realiza la vigilancia de gastroenteritis causadas por rotavirus en niños bajo 5 años a través de centros centinelas ambulatorios y hospitalarios. Objetivo: Conocer la epidemiología de las diarreas por rotavirus y evaluar la carga de morbi-mortalidad. Método: La vigilancia ambulatoria se desarrolla en 14 centros de 8 regiones y la vigilancia hospitalaria se lleva a cabo en 8 hospitales pediátricos de las regiones $\mathrm{V}^{\mathrm{a}}$, VIII ${ }^{\text {a }}$ y RM. Resultados: En 2007, 9,3\% de las consultas por diarrea fue estudiada (339), detectándose rotavirus en $15 \%$ de ellas. Se hospitalizaron por diarrea, 2.074 casos, 9\% del total de las hospitalizaciones en este grupo etario. De estas, 13,6\% fueron por rotavirus. Conclusiones: Dada la situación epidemiológica actual, es importante mantener la vigilancia, ampliar la pesquisa de los casos, aplicar el protocolo de vigilancia establecido y determinar el serotipo/genotipo circulante en el país.

\section{Referencias}

1.- Organización Panamericana de la Salud. Vigilancia epidemiológica de diarreas causadas por rotavirus: guía práctica. Washington, DC. 2007.

2.- Parashar U D, Gibson C J, Bresee J S, Glass R I. Rotavirus and severe childhood diarrhea. Emerg Infect Dis 2006; 12: 304-6.

3.- Gobierno de Chile. Ministerio de Salud de Chile. Departamento de Epidemiología.
Boletín Epidemiológico Mensual BEM. Situación de las enfermedades de notificación obligatoria. 2006; (40): 22-3.

4.- O'Ryan M, Pérez-Schael I, Mamani N, Peña A, Salinas B, González G, et al. Rotavirus-associated medical visits and hospitalizations in South America: A prospective study at three large sentinel hospitals. Pediatr Infect Dis J 2001; 20: 685-93.

5.- Fariñas Reinoso A T. Texto de estudios de postgrado para las ciencias de la salud pública [CD-ROM]. Ciudad de La Habana. Escuela Nacional de Salud Pública. 2005.

6.- Organización Panamericana de la Salud. Las condiciones de Salud en las Américas. Washington, DC. 2000.

7.- Gobierno de Chile. Ministerio de Salud de Chile. División de Rectoría y Regulación Sanitaria, Departamento de Epidemiología. Objetivos sanitarios para la década 20002010. Primera edición, octubre 2002. Pág: 49-50. 